\title{
A Rare Cause of Ortner's Syndrome and a Case-Based Review of Literature
}

\author{
Sukanya Madhuraj ${ }^{1} \cdot$ Harikrishnan Gangadharan $^{1}{ }^{1} \cdot$ Remya Manju $^{1} \cdot$ \\ Akhil Prakash $^{1} \cdot$ Saravana S. Arasan $^{1} \cdot$ Sajitha Krishnadas $^{2} \cdot$ Satish Kumar $^{1}$
}

Received: 17 February 2021 / Accepted: 21 March 2021/Published online: 1 April 2021

(C) Association of Otolaryngologists of India 2021

\begin{abstract}
Ortner's syndrome refers to the compression of the recurrent laryngeal nerve by cardiovascular disorders of various etiologies. We describe a rare case of Ortner's syndrome caused by thoracic aorta aneurysm and a brief review of literature on this unusual cause of Ortner's syndrome. Hoarseness of voice due to the compression of recurrent laryngeal nerve by thoracic aorta aneurysm could be a harbinger of aneurysmal rupture.
\end{abstract}

Keywords Ortner's syndrome · Aortic aneurysm · Cardiovocal syndrome $\cdot$ Recurrent laryngeal nerve palsy

\section{Introduction}

Recurrent laryngeal nerve palsy can be caused by malignant as well as non-malignant lesions [1]. Hoarseness of voice arising from left recurrent laryngeal nerve palsy secondary to mitral stenosis was first described by Norbert Ortner in 1897[2]. Subsequently, similar pathology has been observed in a wide variety of cardiopulmonary disorders and hence grouped as cardiovocal syndrome [3]. Aortic aneurysm has been rarely described to produce Ortner syndrome. We report the case of an elderly gentleman who presented with hoarseness of voice and on further evaluation found to have a thoracic aorta saccular aneurysm with left recurrent laryngeal nerve compression.

Harikrishnan Gangadharan drharikrishnang@gmail.com

1 Department of General Medicine, Government Medical College, Kottayam, Kerala, India

2 Department of Radiodiagnosis, Government Medical College, Kottayam, Kerala, India

\section{Case Report}

A 74 year-old gentleman who was on treatment from elsewhere as a case of chronic obstructive pulmonary disease presented to us with complaints of exertional dyspnea for one year duration and worsening of dyspnea for the last 2 weeks. He had also noticed hoarseness of voice which started 3 months before presenting to us and gradually progressed to inaudible voice at the time of admission at our center. There were multiple episodes of cough during food intake for the last 3 months. There was history of significant weight loss. Though he had orthopnea, he denied any history of chest pain, palpitation, syncope, or episodes of paroxysmal nocturnal dyspnea. Relevant medical history included an angina episode 20 years back which was not evaluated further and the patient was not on any cardiac medication. He was a chronic smoker with 30 pack years and occasionally consumed alcohol.

Clinical examination revealed an emaciated patient with irregularly irregular pulse suggestive of atrial fibrillation with ventricular rate of 88 per minute, bipedal edema, raised jugular venous pressure, cardiomegaly and bi-basal crepitations on auscultation. Provisional diagnosis of acute decompensated heart failure, probable ischemic cardiomyopathy was made. With regards to his hoarseness of voice, the differential diagnosis considered were (1) carcinoma larynx considering his age and smoking history. (2) carcinoma lung with recurrent laryngeal nerve involvement in view of the strong history of smoking and weight loss. (3) laryngeal tuberculosis because of emaciated state with recent significant weight loss.

Laboratory investigations revealed neutrophilic leukocytosis, raised erythrocyte sedimentation rate, C-reactive protein, and transaminitis. COVID-19 RT PCR was negative (Table 1). Electrocardiogram showed atrial fibrillation 
Table 1 Laboratory data of the patient on the day of admission

\begin{tabular}{llc}
\hline Parameter & Patient value & Normal reference range \\
\hline Hemoglobin & $16 \mathrm{~g} / \mathrm{dl}$ & $13.5-17.5 \mathrm{~g} / \mathrm{dl}$ \\
Total leukocyte count & $12930 \mathrm{cells} / \mathrm{mcl}$ & $4000-11,000 \mathrm{cells} / \mathrm{mcl}$ \\
Differential leukocyte count & P83L7M8E2 & $1.5-5.5 \mathrm{lakh}$ \\
Platelet count & $1.21 \mathrm{lakhs}$ & $7-20 \mathrm{mg} / \mathrm{dL}$ \\
Blood urea & $99 \mathrm{mg} / \mathrm{dl}$ & $0.4-1.4 \mathrm{mg} / \mathrm{dl}$ \\
Serum creatinine & $1.4 \mathrm{mg} / \mathrm{dl}$ & $<1 \mathrm{mg} / \mathrm{dl}$ \\
Total Bilirubin & $0.5 \mathrm{mg} / \mathrm{dl}$ & $<0.4 \mathrm{mg} / \mathrm{dl}$ \\
Direct bilirubin & $0.2 \mathrm{mg} / \mathrm{dl}$ & $6-8 \mathrm{~g} / \mathrm{dl}$ \\
Total protein & $5.8 \mathrm{~g} / \mathrm{dl}$ & $3.5-5.5 \mathrm{~g} / \mathrm{dl}$ \\
Serum albumin & $3.3 \mathrm{~g} / \mathrm{dl}$ & $(8-45 \mathrm{U} / \mathrm{L}) /(7-56 \mathrm{U} / \mathrm{L})$ \\
AST/ALT & $250 \mathrm{U} / \mathrm{L} / 477 \mathrm{U} / \mathrm{L}$ & $44-147 \mathrm{IU} / \mathrm{L}$ \\
Serum alkaline phosphatase & $134 \mathrm{IU} / \mathrm{L}$ & $135-145 \mathrm{mEq} / \mathrm{L}$ \\
Serum sodium & $135 \mathrm{mEq} / \mathrm{L}$ & $3.5-5.5 \mathrm{mEq} / \mathrm{L}$ \\
Serum potassium & $4 \mathrm{mEq} / \mathrm{L}$ & $0-22 \mathrm{~mm} / \mathrm{hr}$ \\
ESR & $40 \mathrm{~mm} / \mathrm{h}$ & $0-6 \mathrm{mg} / \mathrm{L}$
\end{tabular}

g/dl-gram per decilitre,mcl-microlitre,AST-Aspartate amino transferase,ALT-Alanine amino transferase,IU/L-international units per litre,U/LUnits per litre,mEq/L-milli equivalents per litre,mm/h-millimeter per hour,mg/L-milli gram per litre

and no signs of ischemia. Chest X-ray showed superior mediastinal widening, cardiomegaly and bilateral patchy infiltrates (Fig. 1a). 2D Echocardiography showed features of global left ventricular hypokinesia and severe left ventricular dysfunction with moderate tricuspid regurgitation, mild pulmonary artery hypertension and an ejection fraction of $38 \%$. A lower respiratory tract infection precipitating cardiac failure, congestive hepatitis and probable chronic aspiration pneumonitis was considered. As he had hoarseness with superior mediastinal widening otorhinolaryngology examination evaluation was sought. Indirect laryngoscopy revealed left vocal cord palsy. Contrast enhanced CT thorax revealed cardiomegaly, thrombosed saccular aneurysmal dilatation of the proximal descending thoracic aorta arising next to origin of left subclavian artery with a length of $5.1 \mathrm{~cm}$ and height $2.2 \mathrm{~cm}$ with eccentric thrombus (Fig. 1b). Hence a diagnosis of Ortner's syndrome due to saccular aneurysmal dilatation of the proximal descending thoracic aorta compressing left recurrent laryngeal nerve causing hoarseness
Fig. 1 a Chest $X$ ray PA view showing superior mediastinal widening with tracheal deviation to right, hyperinflated lung fields with bilateral infiltrates and cardiomegaly. b Transverse contrast enhanced CT Thorax showing aortic saccular aneurysm of descending aorta just distal to left subclavian and protruding into aorto-pulmonary window.
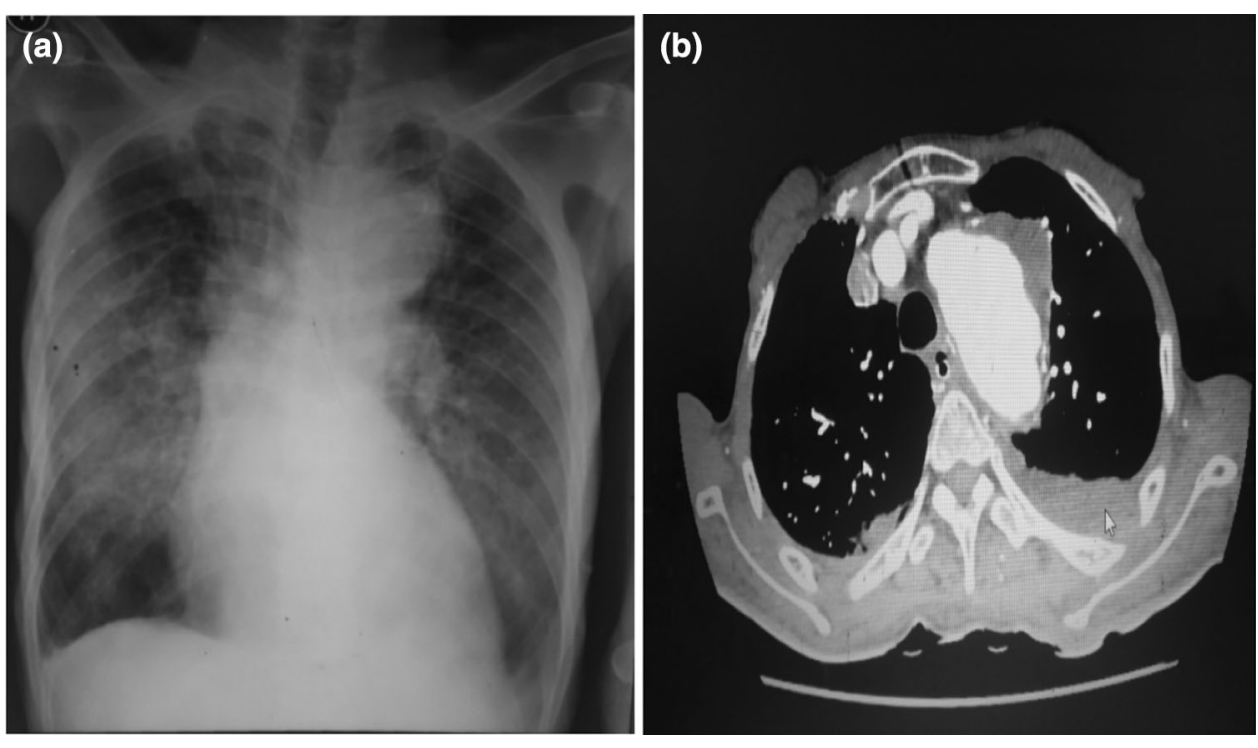
of voice was made. He was managed with intravenous broad spectrum antibiotics, diuretics, and other supportive measures. His cardiac failure improved and aortic aneurysm repair was suggested. However due to financial constraints and the high risk associated with procedure, he refused surgical intervention. He succumbed to hospital acquired pneumonia after 3 weeks of in-patient care at our center.

\section{Discussion}

Left recurrent laryngeal nerve is particularly vulnerable to compression compared to its counterpart on right due to its long circuitous course and proximity to major mediastinal vessels, trachea, esophagus, and lung apex. Loughran et al. studied 77 patients prospectively at a Scottish center and found that the most common cause of unilateral recurrent laryngeal nerve palsy was carcinoma lungs (43\%). Idiopathic causes including Ortner's syndrome contributed to $11 \%$ of causes [4]. In a systematic review of cardiovocal syndrome done by Mulpuru et al. [5], causes of Ortner syndrome included mitral stenosis, mitral regurgitation, left ventricular failure, pulmonary hypertension, atrial septal defect, ventricular septal defect, and Eisenmenger's complex. Aortic aneurysms which can be saccular, atherosclerotic, traumatic, or mycotic can lead to Ortner's syndrome. Five percent of cases of recurrent laryngeal nerve palsy is due to thoracic aortic aneurysm [6].

Different mechanisms have been put forward to explain recurrent laryngeal nerve palsy in Ortner's syndrome. Initially, Ortner attributed the compression of the left recurrent laryngeal nerve by the dilated left atrium against the arch of aorta to be responsible for vocal cord palsy. Autopsy studies have shown that compression of recurrent laryngeal nerve occurs between the aorta and pulmonary artery near the ligamentum arteriosum [7].

Teixido et al. studied 168 cases of thoracic aorta aneurysm retrospectively and found that chest or back pain is the most frequent initial symptom and hoarseness of voice was found as the initial symptom in only 5\% of cases. Type 1 and type 3 aneurysm occurred with almost similar frequency in this series and type 2 aneurysm was uncommon. Postoperative vocal cord dysfunction developed in $12 \%$ of patients [6]. Hoarseness indicates significant aneurysmal dilation and has been pointed out by many authors as a harbinger of imminent aneurysmal rupture. Okhi M [8] reported 2 patients with left recurrent laryngeal nerve palsy caused by thoracic aorta saccular aneurysm. While the first patient developed aneurysmal rupture one month after the onset of hoarseness of voice requiring emergency aortic arch replacement, the second patient was managed by elective aortic arch replacement surgery.
Lydakis et al. [9] reported the case of a 86 year old woman with hoarseness of voice as the only major clinical symptom of a large thoracic aorta aneurysm which ruptured soon after presentation. In a case series of 7 patients with thoracic aorta aneurysm causing recurrent laryngeal nerve palsy described by Ismazizi et al. [10], all patients had hoarseness of voice as their first symptom. 4 patients had dysphagia due to oesophageal compression along with hoarseness of voice. Most of the aneurysms were involving the arch and proximal descending thoracic aorta. Out of the 7 patients, four had saccular type of aortic aneurysm.

Ismazizi et al. [10] reported improvement in hoarseness in four out of five patient who underwent thoracic endovascular aneurysm repair (TEVAR). In patients who are at high risk for open repair of aneurysm, TEVAR offers a less invasive, lower operative risk procedure. Ishii et al. [11] determined the relationship between outcome of recurrent laryngeal nerve palsy and the surgical procedure employed. Out of the five patients who had non-surgical paralysis (due to thoracic aorta aneurysm per se) four underwent aortic replacement. Hoarseness and aspiration improved with regaining of vocal cord mobility in two, two patients developed atrophy of the vocal cord with exacerbation of aspiration and hoarseness of voice. The patient who did not undergo vessel replacement had gradual worsening of symptoms on follow up. The authors concluded that once recurrent laryngeal nerve palsy has occurred, aggressive intervention with rehabilitation and surgical management should be considered rather than mere observation.

To conclude thoracic aorta aneurysm can present with Ortner's syndrome as the first and only manifestation. Hoarseness of voice may be a sign of impending aneurysmal rupture and poor prognosis. It is of paramount importance that an evaluation for an aortic aneurysm is done in any patient presenting with new-onset hoarseness.

Authors' Contributions HG and SK contributed to the conception and design of the work. All authors contributed to the acquisition of data for the work. All authors contributed to drafting of the work. All authors contributed in final approval of the version to be published. All authors agree to be accountable for all aspects of the work in ensuring that questions related to the accuracy or integrity of any part of the work are appropriately investigated and resolved.

\section{Declarations}

Conflict of interest The authors declare that they have no conflict of interest.

Informed Consent We obtained informed consent from the patient including consent for publication. 


\section{References}

1. Yumoto E, Minoda R, Hyodo M et al (2002) Causes of recurrent laryngeal nerve paralysis. Auris Nasus Larynx 29(1):41-45. https://doi.org/10.1016/s0385-8146(01)00122-5

2. Ortner N (1897) Recurrenslähmung bei Mitralstenose. Wien Klin Wochenschr 10:753-755

3. Plastiras SC, Pamboucas C, Zafiriou T et al (2010) Ortner's syndrome: a multifactorial cardiovocal syndrome. Clin Cardiol 33(6):E99-100. https://doi.org/10.1002/clc.20646

4. Loughran S, Alves C, MacGregor FB (2002) Current aetiology of unilateral vocal fold paralysis in a teaching hospital in the West of Scotland. J Laryngol Otol 116(11):907-910. https://doi.org/10.1258/00222150260369426

5. Mulpuru SK, Vasavada BC, Punukollu GK et al (2008) Cardiovocal syndrome: a systematic review. Heart Lung Circ 17(1):1-4

6. Teixido MT, Leonetti JP (1990) Recurrent laryngeal nerve paralysis associated with thoracic aortic aneurysm. OtolaryngolHead Neck Surg 102(2):140-144
7. Fetterolf G, Norris G (1911) The anatomical explanation of paralysis of left recurrent laryngeal nerve found in certain case of mitral stenosis. Am J Med Sci 141:625-638

8. Ohki M (2012) Thoracic saccular aortic aneurysm presenting with recurrent laryngeal nerve palsy prior to aneurysm rupture: a prodrome of thoracic aneurysm rupture? Case Rep Otolaryngol 2012:367873

9. Lydakis C, Thalassinos E, Apostolakis S et al (2006) Hoarseness as imminent symptom of aortic aneurysm rupture (Ortner's syndrome). Int Angiol 25(2):231-233

10. Ismazizi Z, Zainal AA (2016) Thoracic Aortic Aneurysm as A Cause of Ortner's Syndrome - A Case Series. Med J Malaysia 71(3):139-141

11. Ishii K, Adachi H, Tsubaki K et al (2004) Evaluation of recurrent nerve paralysis due to thoracic aortic aneurysm and aneurysm repair. Laryngoscope 114(12):2176-2181

Publisher's Note Springer Nature remains neutral with regard to jurisdictional claims in published maps and institutional affiliations. 